\title{
Effectiveness and safety of common therapeutic drugs for refractory lupus nephritis: A network meta-analysis
}

\author{
JUN ZHOU ${ }^{1}$, MENG-JUN TAO ${ }^{1}$, LAI-RUN JIN ${ }^{1}$, JUN SHENG ${ }^{2}$, ZHI LI $^{2}$, HUI PENG $^{3}$, LIANG XU $^{2}$ and HUI YUAN ${ }^{1}$ \\ ${ }^{1}$ Department of Epidemiology and Biostatistics, School of Public Health, Wannan Medical College, \\ Wuhu, Anhui 241002; ${ }^{2}$ Department of Rheumatology and ${ }^{3}$ Hospital Infection Control Office, \\ Affiliated Yijishan Hospital of Wannan Medical College, Wuhu, Anhui 241001, P.R. China
}

Received May 31, 2019; Accepted November 6, 2019

DOI: $10.3892 /$ etm.2019.8257

\begin{abstract}
Previous studies have indicated that various drugs may be beneficial for the treatment of patients with refractory lupus nephritis (RLN). The present study aimed to evaluate the effectiveness and safety of common therapeutic drugs for the treatment of RLN using a network meta-analysis (NMA). NMA was performed using Stata 14.0 software. The odds ratio (OR) and 95\% CI were calculated. A total of 19 studies comprising 1,127 patients were included. Common therapeutic drugs for RLN included glucocorticoids (GC), cyclophosphamide (CTX), mycophenolate mofetil (MMF), tacrolimus (TAC), leflunomide (LEF), cyclosporine A and rituximab (RTX). Evaluation of the effectiveness revealed that $\mathrm{MMF}+\mathrm{GC}$ produced significantly higher overall responses (i.e. complete remission plus partial remission) and that $\mathrm{MMF}$ $+\mathrm{GC}(\mathrm{OR}=2.58 ; 95 \% \mathrm{CI}, 1.67-3.97), \mathrm{CTX}+\mathrm{RTX}+\mathrm{GC}$ $(\mathrm{OR}=3.89 ; 95 \% \mathrm{CI}, 1.60-9.45), \mathrm{CTX}+\mathrm{LEF}+\mathrm{GC}(\mathrm{OR}=3.05$; $95 \% \mathrm{CI}, 1.05-8.84)$ and $\mathrm{CTX}+\mathrm{TAC}+\mathrm{GC}(\mathrm{OR}=6.22 ; 95 \%$ CI, 1.93-20.05) had significantly higher overall responses compared with those to the traditional treatment regimen $(\mathrm{CTX}+\mathrm{GC})$. Ranking probability based on the surface under the cumulative ranking curve indicated that CTX $+\mathrm{TAC}+\mathrm{GC}$ had the highest probability $(80.6 \%)$ of being the best treatment for achieving an overall response. In the safety evaluation, $\mathrm{MMF}+\mathrm{GC}$ had a lower risk of infection than CTX + GC $(\mathrm{OR}=0.32 ; 95 \% \mathrm{CI}, 0.11,0.88)$. There were no statistically
\end{abstract}

Correspondence to: Dr Hui Yuan, Department of Epidemiology and Biostatistics, School of Public Health, Wannan Medical College, 22 Wenchangxi Road, Wuhu, Anhui 241002, P.R. China

E-mail: yuanhui0553@126.com

Abbreviations: SLE, systemic lupus erythematosus; RLN, refractory lupus nephritis; NMA, network meta-analysis; GC, glucocorticoids; CTX, cyclophosphamide; MMF, mycophenolate mofetil; TAC, tacrolimus; LEF, leflunomide; CsA, cyclosporine A; RTX, rituximab; SUCRA, surface under the cumulative ranking curve; $\mathrm{CR}$, complete remission; $\mathrm{PR}$, partial remission

Key words: refractory lupus nephritis, cyclophosphamide, mycophenolate mofetil, tacrolimus, network meta-analysis significant differences in adverse reactions, including gastrointestinal reactions and leukopenia between any two treatment regimens. In conclusion, the regimen of CTX $+\mathrm{TAC}+\mathrm{GC}$ exhibited a trend in superiority regarding clinical efficacy among common therapeutic drug treatments for RLN, while the regimen of CTX $+\mathrm{GC}$ had a higher probability to cause adverse effects among the nine interventions compared.

\section{Introduction}

Lupus nephritis (LN) is one of the most common clinical manifestations and serious complications in patients with systemic lupus erythematosus (SLE). Almost all patients with SLE have renal damage in the course of their disease and $25-50 \%$ of patients have clinical manifestations of nephropathy at the time of diagnosis (1). In recent years, due to early diagnosis and comprehensive treatment, the survival rate of patients with LN has significantly improved, but in certain patients, traditional immunosuppressive agents are still ineffective. Indeed, $\sim 30 \%$ of treated patients fail to improve and certain patients develop refractory LN (RLN) after one year of traditional treatment (2).

Patients with RLN have been indicated to display no serum creatinine improvement after 2-3 months under the traditional treatment regimen [cyclophosphamide (CTX) + glucocorticoids (GC)] or no proteinuria or albumin improvement after 6 months $(3,4)$. According to the World Health Organization, LN may be classified into 6 categories based on renal biopsy, ranging from type I to type VI and the respective prognosis is in the order from good to bad. In general, patients with class III, IV, V, III+V and IV+V LN are prone to develop RLN. A cohort study in Egypt indicated that patients with class II LN had a complete response to treatment and $27.3 \%$ of patients with class III, IV and V LN did not respond to treatment (5). Patients with LN who do not respond to treatment tend to have more irreversible injuries and the condition is more likely to deteriorate in such patients. A cohort study from a Chinese population suggested that the treatment was ineffective in $\sim 26.5 \%$ of the population and they eventually developed end-stage renal failure (6). Another study suggested that failure to respond after treatment for one year was an independent predictor of mortality in patients with LN (7). 
If the traditional treatment regimen fails, such as if there is no response after 6 months of treatment, the patient has refractory $\mathrm{LN}$ (8). In this case, a large number of novel pharmaceutical preparations, including mycophenolate mofetil (MMF), tacrolimus (TAC) and rituximab (RTX), have been developed in China and elsewhere, and these drugs were reported to have a certain therapeutic effect in RLN. However, there are no studies that evaluate the best treatment for RLN. Therefore, the present meta-analysis study aimed to systematically evaluate the effectiveness and safety of common therapeutic drugs for the treatment of RLN and screen the optimal therapeutic drugs or regimens to provide a basis for the clinical treatment of RLN.

\section{Materials and methods}

Search strategy. The PubMed, Embase, Cochrane Library, China Knowledge Network Infrastructure, VIP and Wanfang databases were searched from inception to April 30, 2018. The search terms include 'refractory', 'lupus nephritis' and 'treatment'. The search strategy was adjusted according to the different databases. The title and abstract of the citations were screened first, and then the full text was screened. In addition, the references were manually checked in published reviews to thoroughly search the literature.

Inclusion and exclusion criteria. The following publication references were included in the present study: i) Subjects, patients with refractory lupus nephritis; ii) randomized controlled trials (RCTs); iii) observation group or control group containing $\geq 1$ of the following drugs: Glucocorticoids, cyclophosphamide, mycophenolate mofetil, tacrolimus, leflunomide, cyclosporine A and/or rituximab; and iv) available data, effectiveness or adverse reactions. However, studies on animal models, crossover studies, case reports and studies without sufficient data were excluded.

Data extraction and quality assessment. Then, two reviewers independently screened and crosschecked the literature. In case of disagreement, the discussion was resolved or submitted to a third researcher. Duplicates were removed, and unrelated literature was excluded by reading the title and abstract. Then, the citations were determined by reading the full text. The collected data mainly included indicators of effectiveness i.e. complete remission (CR) and partial remission (PR); evaluation criteria for effectiveness, i.e. $24-\mathrm{h}$ urine protein $<0.3 \mathrm{~g} / \mathrm{l}$ for CR, and 24-h urine protein $<1.0 \mathrm{~g} / 1$, serum albumin $>30 \mathrm{~g} / 1$ and serum creatinine $<25 \%$ for PR; the total number of cases with effective treatment was determined as the number of cases with CR + number of cases with PR (9). The safety indicators were infection and other adverse reactions including gastrointestinal reactions, leukopenia, liver damage and bone marrow suppression. The Jadad scoring standard was used to quantitatively evaluate the quality of the included studies. The score ranged from 0 to 5 ; a total score of $\geq 4$ indicated high quality (10)

Statistical analysis. Stata 14.0 software (Stata Corp.) was used in the present Network Meta Analysis (NMA). The odds ratio and $95 \% \mathrm{CI}$ were determined to compare the efficacy and adverse reactions of the nine treatment regimens for RLN. The degree of heterogeneity was quantified using $\mathrm{I}^{2}$ calculations. Values of $\mathrm{I}^{2}<50 \%$ were considered to indicate no heterogeneity. The analysis was performed using the fixed-effects model or random-effects model as appropriate. Furthermore, a sensitivity analysis was performed by omitting studies one by one to determine the source of heterogeneity. The P-value of the $\mathrm{Z}$ test was used to assess the direct and indirect comparison results in NMA (11). If $\mathrm{P}>0.05$, the direct comparison result is consistent with the indirect comparison result; otherwise it is inconsistent. The efficacy and adverse reaction risks of each treatment regimen were sequenced using the surface under the cumulative ranking curve (SUCRA) and the optimal treatment protocol was determined.

\section{Results}

Selection of studies for inclusion. A total of 1,366 relevant articles were initially retrieved, of which 494 duplicated articles and 819 studies (87 reviews, 112 animal experiments and 620 non-randomized controlled trials) were excluded after reading their titles and abstracts. In addition, 34 articles did not meet the inclusion criteria and were also excluded. Finally, 19 articles comprising a total of 1,127 patients were included (Fig. 1).

Studies included in the meta-analysis. Among the 19 articles, 17 were in Chinese $(9,12-27)$ and 2 were in English $(28,29)$. Except for one study that was a three-arm study (26), the remaining 18 were two-arm studies. Only three studies $(7,21,25)$ were identified as high-quality studies (Jadad score=4). A total of seven therapeutic drugs were used in nine different treatment regimes. The basic characteristics of the studies included and the treatment regimens are specified in Table I. Fig. 2 shows the sample size for each treatment regimen and the number of studies compared between the two regimens, such as the largest number of studies comparing CTX + GC and $\mathrm{MMF}+\mathrm{GC}$.

Analysis of inconsistency. In the evidence network diagram, $\mathrm{CTX}+\mathrm{GC}, \mathrm{MMF}+\mathrm{GC}$ and $\mathrm{CTX}+\mathrm{TAC}+\mathrm{GC}$ formed a closed loop; thus, the direct evidence and indirect evidence of the three treatment regimens were consistently tested. The $Z$ test result was indicated no inconsistency $(\mathrm{P}=0.931, \mathrm{P}>0.05)$.

Detection of publication bias. A funnel plot was used to evaluate publication bias. The funnel plot was symmetrical in this analysis, and it can be considered that there was no publication bias in the direct comparison meta-analysis (Fig. 3).

\section{NMA results}

Comparison of treatment effect. The present results suggested that $\mathrm{MMF}+\mathrm{GC}$ produced significantly higher overall responses $(\mathrm{CR}+\mathrm{PR})$, and $\mathrm{MMF}+\mathrm{GC}(\mathrm{OR}=2.58 ; 95 \% \mathrm{CI}, 1.67-3.97)$, $\mathrm{TAC}+\mathrm{GC}(\mathrm{OR}=3.14 ; 95 \% \mathrm{CI}, 1.05-9.43), \mathrm{CTX}+\mathrm{LEF}+\mathrm{GC}$ $(\mathrm{OR}=3.05 ; 95 \% \mathrm{CI}, 1.05-8.84), \mathrm{CTX}+\mathrm{RTX}+\mathrm{GC}(\mathrm{OR}=3.89$; 95\% CI, 1.60-9.45) and CTX+TAC+GC $(\mathrm{OR}=6.22 ; 95 \% \mathrm{CI}$, 1.93-20.05) had significantly higher overall responses than $\mathrm{CTX}+\mathrm{GC}$. In addition, the present results suggested that 


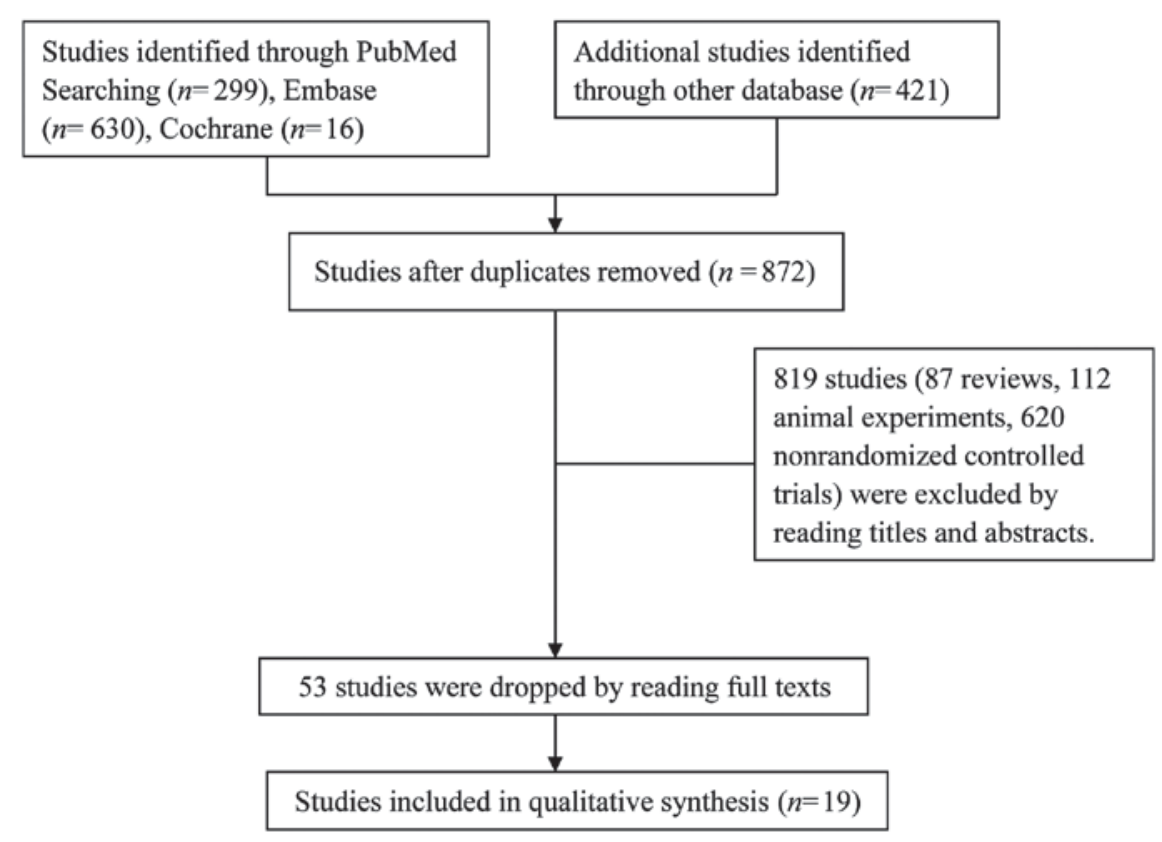

Figure 1. Flow diagram of the selection of included studies.

$\mathrm{MMF}+\mathrm{GC}$ produced significantly higher overall responses, and $\mathrm{MMF}+\mathrm{GC}(\mathrm{OR}=4.13 ; 95 \% \mathrm{CI}, 1.72-9.96)$, TAC $+\mathrm{GC}$ $(\mathrm{OR}=5.04 ; 95 \% \mathrm{CI}, 1.16-21.97), \mathrm{CTX}+\mathrm{LEF}+\mathrm{GC}(\mathrm{OR}=4.89$; $95 \% \mathrm{CI}, 1.15-20.78), \mathrm{CTX}+\mathrm{RTX}+\mathrm{GC} \quad(\mathrm{OR}=6.24 ; 95 \%$ $\mathrm{CI}, 1.66-23.39)$ and $\mathrm{CTX}+\mathrm{TAC}+\mathrm{GC}(\mathrm{OR}=9.98 ; 95 \% \mathrm{CI}$, 2.23-44.56) had significantly higher overall responses than GC. There were no statistically significant differences in the effectiveness of any of the other regimens in the treatment of RLN (Fig. 4).

Comparison of safety. Comparison of infection suggested that the difference between $\mathrm{MMF}+\mathrm{GC}$ and $\mathrm{CTX}+\mathrm{GC}$ was statistically significant. Comparison of gastrointestinal reactions and leukopenia indicated no statistically significant differences between any of the groups (Figs. 5 and 6).

Results sorting. In terms of efficiency, the maximum SUCRA value of CTX+TAC+GC was $80.6 \%$, indicating that TAC+CTX+GC was likely to be the best currently used treatment. The maximum SUCRA values of CTX+GC were $67.8 \%$, $80.1 \%$ and $65.4 \%$ for infection, gastrointestinal reaction and leukopenia, indicating that $\mathrm{CTX}+\mathrm{GC}$ had the highest risk of adverse reaction (Table II).

\section{Discussion}

At present, the cause of RLN remains elusive, but studies have indicated that non-compliance with treatment and lack of efficacy of the induction therapy regimens for LN may promote the development of RLN (7). However, there is no uniform guideline for the treatment of RLN patients; therapeutic drugs and treatment regimens depend largely on the patients' condition and the clinician's experience. Therefore, the present study compared the effectiveness and safety of common therapeutic drugs for the treatment of RLN to provide a reference for the clinical prescription of drugs.
The traditional induction therapy used for LN is a combination of GC and intravenous CTX. The basic drug used in the induction phase is CTX at a sufficient dose, with a subsequent gradual dose reduction according to the patient's condition. In order to avoid the toxic effects of CTX, it recently was replaced with MMF $(30,31)$. Studies have indicated that, although the effectiveness of MMF in the treatment of $\mathrm{LN}$ is not different from that of CTX, it reduces the risk of ovarian failure associated with CTX (32). However, the present study indicated that MMF was more effective and was associated with less gastrointestinal adverse reactions than CTX in the treatment of RLN. In addition, MMF had a greater likelihood of reducing the risk of adverse effects, including infection and leukopenia, when compared with CTX.

The present study also indicated that TAC combined with CTX in the treatment of RLN was superior to the traditional regimen, but did not perform differently from MMF. This is consistent with previous results from an NMA of three drugs for LN (33). However, these three treatment regimens exhibited no difference in adverse reactions, including infection, leukopenia and gastrointestinal reactions. The mechanisms of action of MMF and tacrolimus are different; MMF reduces B-cell and T-cell proliferation, leads to antibody production, and recruitment of lymphocytes and monocytes, while tacrolimus reduces cytokine production and T-cell activation, and stabilizes the actin cytoskeleton of podocytes (34). Although the two drugs produce similar effects, tacrolimus is more expensive. RLN patients require long-term medication, and thus, the patients' financial situation should also be considered when selecting an effective treatment.

In a prospective cohort study (35), a multi-target regimen (MMF+TAC+GC) was first proposed for the treatment of $\mathrm{LN}$, and the incidence of complete remission were significantly higher than those with intravenous CTX, with fewer adverse events. The multi-target treatment also exhibited certain advantages in the treatment of RLN with not only a better 
Table I. Basic characteristics of the studies included.

\begin{tabular}{|c|c|c|c|c|c|c|c|}
\hline $\begin{array}{l}\text { First } \\
\text { author (year) }\end{array}$ & $\begin{array}{l}\text { Sample } \\
\text { size }(n)\end{array}$ & $\mathrm{M} / \mathrm{F}$ & Treatment regimen & $\begin{array}{l}\text { Biopsy } \\
\text { class }\end{array}$ & $\begin{array}{l}\text { Duration } \\
\text { (months) }\end{array}$ & $\begin{array}{l}\text { Jadad } \\
\text { score }\end{array}$ & (Refs.) \\
\hline Ma (2016) & 64 & $40 / 24$ & GC, MMF (25 mg/day)+GC & Unclear & 3 & 3 & $(9)$ \\
\hline Li (2012) & 86 & $49 / 37$ & $\mathrm{GC}, \mathrm{MMF}(25 \mathrm{mg} /$ day $)+\mathrm{GC}$ & Unclear & 3 & 3 & $(10)$ \\
\hline Wang (2015) & 156 & $20 / 136$ & CTX (100 mg/m²)+GC, MMF (1.5 g/day $)+G C$ & Unclear & 6 & 3 & $(11)$ \\
\hline Zheng (2015) & 31 & $18 / 13$ & $\mathrm{CTX}+\mathrm{GC}, \mathrm{MMF}(0.1 \mathrm{~g} /$ day $)+\mathrm{GC}$ & Unclear & 3 & 2 & $(12)$ \\
\hline Dong (2014) & 78 & $9 / 69$ & $\operatorname{CTX}\left(1,000 \mathrm{mg} / \mathrm{m}^{2}\right)+\mathrm{GC}, \mathrm{MMF}(1.5 \mathrm{~g} /$ day $)+\mathrm{GC}$ & Unclear & 24 & 3 & (13) \\
\hline Liu (2013) & 79 & $9 / 70$ & CTX $\left(100 \mathrm{mg} / \mathrm{m}^{2}\right)+\mathrm{GC}, \mathrm{MMF}(1.5 \mathrm{~g} /$ day $)+\mathrm{GC}$ & III, IV,V & 24 & 3 & $(14)$ \\
\hline Liu (2009) & 39 & $7 / 32$ & CTX (100 mg/m²)+GC, MMF (1.5 g/day)+GC & IV & 24 & 3 & $(15)$ \\
\hline Yang (2008) & 60 & $22 / 38$ & $\mathrm{CTX}\left(200 \mathrm{mg} / \mathrm{m}^{2}\right)+\mathrm{GC}, \mathrm{MMF}(0.75 \mathrm{mg} /$ day $)+\mathrm{GC}$ & Unclear & 6 & 3 & $(16)$ \\
\hline Zhen (2006) & 40 & $26 / 14$ & CTX $\left(1,000 \mathrm{mg} / \mathrm{m}^{2}\right)+\mathrm{GC}, \mathrm{MMF}(1.5 \mathrm{~g} /$ day $)+\mathrm{GC}$ & Unclear & 6 & 3 & $(17)$ \\
\hline Shi (2006) & 53 & $5 / 48$ & CTX (400 mg/m²)+GC, MM F(1.5 g/day)+GC & IV & 6 & 3 & $(18)$ \\
\hline Li (2002) & 40 & $1 / 39$ & CTX $\left(400 \mathrm{mg} / \mathrm{m}^{2}\right)+\mathrm{GC}, \mathrm{MMF}(1.5 \mathrm{~g} /$ day $)+\mathrm{GC}$ & Unclear & 3 & 3 & (19) \\
\hline Mostafi (2010) & 31 & $4 / 27$ & CsA (4 mg/kg/day)+GC, MMF (1.5 g/day)+GC & IV & 108 & 3 & $(20)$ \\
\hline Lu (2017) & 74 & $25 / 49$ & $\operatorname{CTX}\left(1,000 \mathrm{mg} / \mathrm{m}^{2}\right)+\mathrm{GC}, \mathrm{TAC}(2 \mathrm{mg} /$ day $)+\mathrm{GC}$ & Unclear & 12 & 4 & $(21)$ \\
\hline Liu (2016) & 30 & $3 / 27$ & LE F(30 mg/day)+GC, TAC (2 mg/day)+GC & $\begin{array}{l}\text { III, IV, V, } \\
\text { III }+V, I V+V\end{array}$ & 6 & 3 & $(22)$ \\
\hline Xiang (2017) & 60 & $7 / 53$ & $\begin{array}{l}\text { CTX }\left(400 \mathrm{mg} / \mathrm{m}^{2}\right)+\mathrm{GC}, \mathrm{CTX}\left(400 \mathrm{mg} / \mathrm{m}^{2}\right)+ \\
\mathrm{LEF}(20 \mathrm{mg} / \text { day })+\mathrm{GC}\end{array}$ & IV & 6 & 4 & (7) \\
\hline Liang (2014) & 50 & $13 / 37$ & $\begin{array}{l}\text { CTX }\left(400 \mathrm{mg} / \mathrm{m}^{2}\right)+\mathrm{GC}, \text { CTX }\left(400 \mathrm{mg} / \mathrm{m}^{2}\right)+ \\
\text { TAC }(3.5 \mathrm{mg} / \text { day })+\mathrm{GC}\end{array}$ & Unclear & 6 & 3 & (23) \\
\hline Yi (2014) & 27 & $5 / 22$ & $\begin{array}{l}\operatorname{CTX}\left(800 \mathrm{mg} / \mathrm{m}^{2}\right)+\mathrm{GC}, \mathrm{CTX}\left(800 \mathrm{mg} / \mathrm{m}^{2}\right)+ \\
\operatorname{RTX}\left(375 \mathrm{mg} / \mathrm{m}^{2}\right)+\mathrm{GC}\end{array}$ & $\mathrm{III}+\mathrm{V}, \mathrm{IV}+\mathrm{V}$ & 6 & 3 & $(24)$ \\
\hline Zhang (2015) & 84 & - & $\begin{array}{l}\text { CTX }\left(800 \mathrm{mg} / \mathrm{m}^{2}\right)+\mathrm{GC}, \text { CTX }\left(800 \mathrm{mg} / \mathrm{m}^{2}\right)+ \\
\operatorname{RTX}\left(375 \mathrm{mg} / \mathrm{m}^{2}\right)+\mathrm{GC}\end{array}$ & $\mathrm{III}+\mathrm{V}, \mathrm{IV}+\mathrm{V}$ & 12 & 4 & $(25)$ \\
\hline Mo (2009) & 45 & $4 / 41$ & $\begin{array}{l}\text { CTX }\left(400 \mathrm{mg} / \mathrm{m}^{2}\right)+\mathrm{GC} \text { MMF }(1.5 \mathrm{~g} / \text { day })+\mathrm{GC} \\
\text { CTX }\left(400 \mathrm{mg} / \mathrm{m}^{2}\right)+\mathrm{TAC}(3.5 \mathrm{mg} / \text { day })+\mathrm{GC}\end{array}$ & $\begin{array}{l}\mathrm{IV}, \mathrm{V}, \mathrm{III}+\mathrm{V} \\
\mathrm{IV}+\mathrm{V}\end{array}$ & 6 & 2 & (26) \\
\hline
\end{tabular}

M/F, male/female; GC, glucocorticoid; CTX, cyclophosphamide; MMF, mycophenolate; TAC, tacrolimus; LEF, leflunomide; RTX, rituximab; CsA, cyclosporine A; d, day.

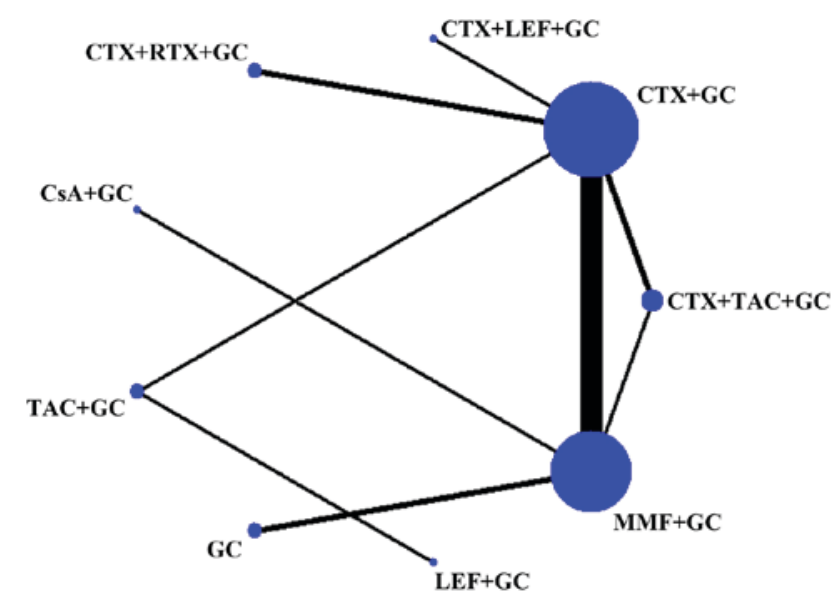

Figure 2. Network diagram of evidence for treatment efficacy of each regimen. The size of each node is proportional to the sample size of the individual treatment regimen; the widths of the connecting lines are proportional to the number of studies compared between the two regimens. GC, glucocorticoid; CTX, cyclophosphamide; MMF, mycophenolate; TAC, tacrolimus; LEF, leflunomide; RTX, rituximab; CsA, cyclosporine A.

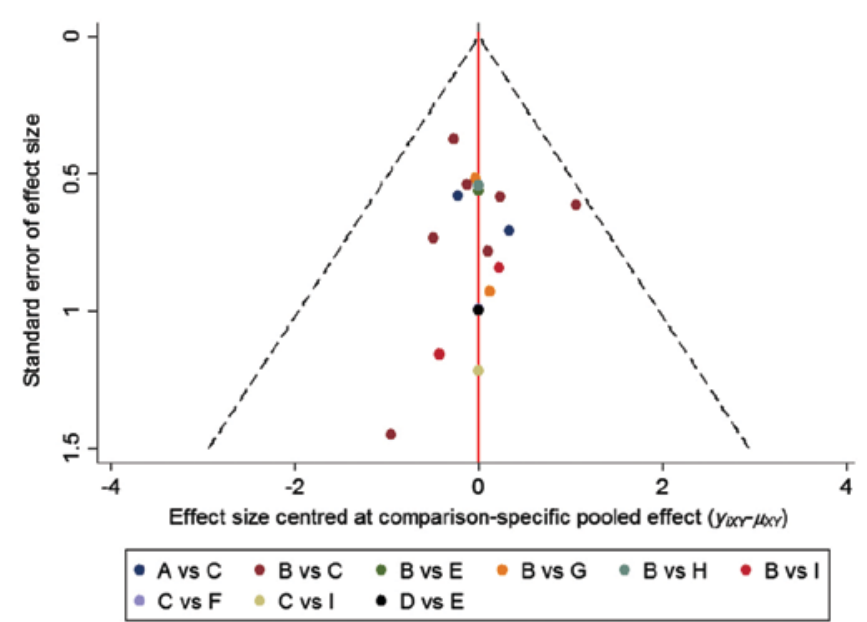

Figure 3. Funnel plot for assessment of publication bias or small sample effect. A, GC; B, CTX+GC; C, MMF+GC; D, LEF+GC; E, FK506+GC; F, CsA+GC; G, CTX+RTX+GC; H, CTX+LEF+GC; I, CTX+FK506+GC. GC, glucocorticoid; CTX, cyclophosphamide; MMF, mycophenolate; TAC, tacrolimus; LEF, leflunomide; RTX, rituximab; CsA, cyclosporine A. 


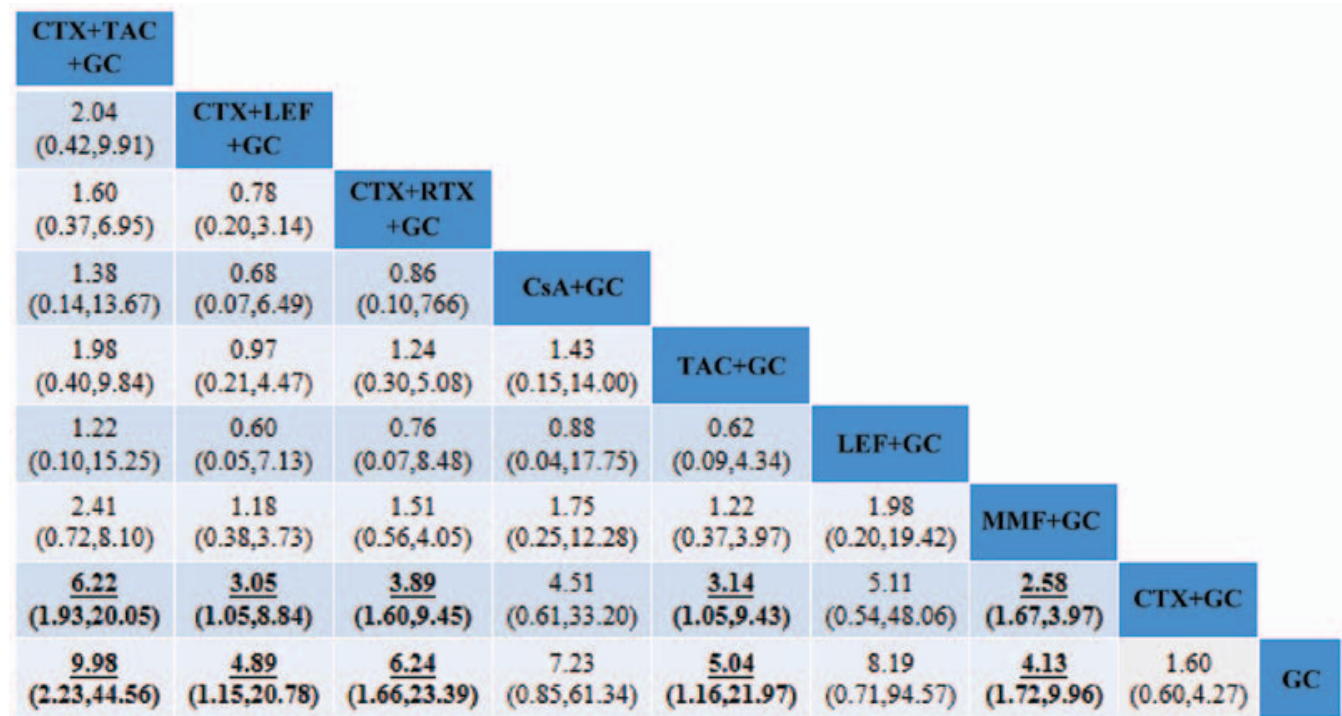

Figure 4. Comparison of the therapeutic effects of the regimens. The OR and 95\% CI for comparison of the efficacy of each treatment regimen are provided. The results of the plots are read from top to bottom and from left to right. An OR $>1$ indicates that the treatment on the top left is better than the comparative treatment. For instance, the effectiveness of TAC+CTX+GC is better than that of CTX+GC. The underlined and bold numbers indicate statistical significance. OR, odds ratio; GC, glucocorticoid; CTX, cyclophosphamide; MMF, mycophenolate; TAC, tacrolimus; LEF, leflunomide; RTX, rituximab; CsA, cyclosporine A.

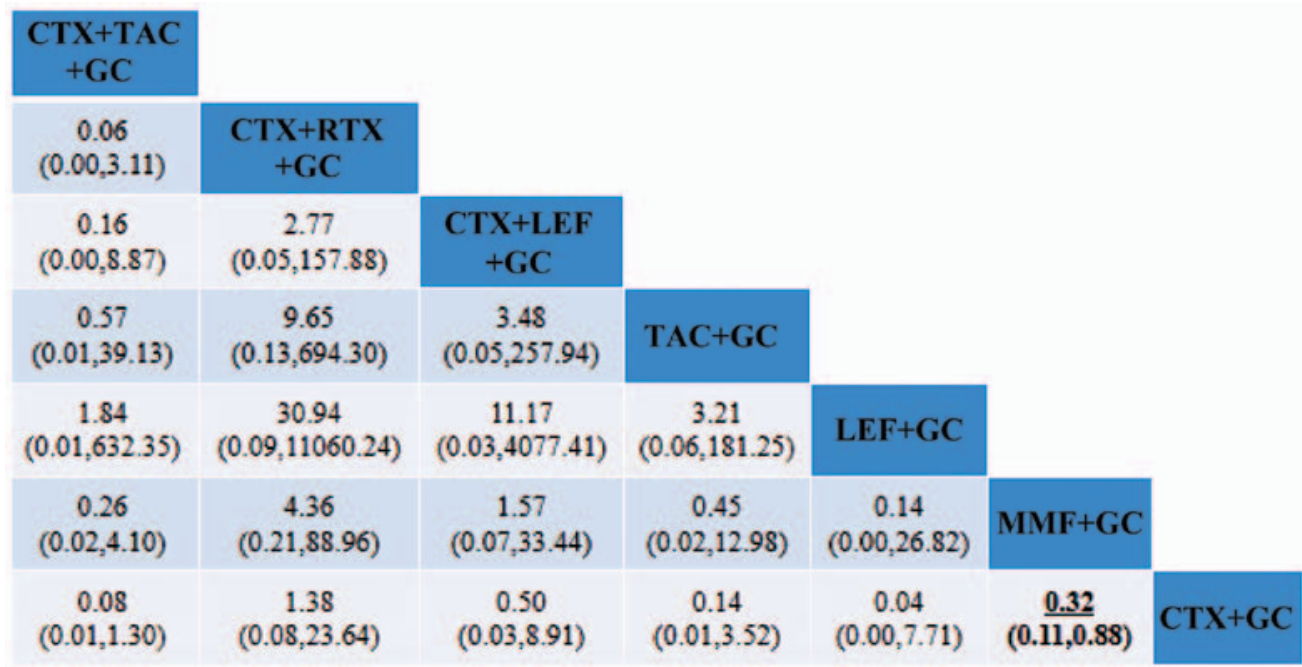

figure 5. Comparison of infections after treatment according to each regimen. An OR $<1$ means that the possibility of infection after treatment on the top left was lower than that after the comparative treatment. For instance, MMF+GC treatment of RLN had a lower risk of infection than CTX+GC. OR, odds ratio; GC, glucocorticoid; CTX, cyclophosphamide; MMF, mycophenolate; TAC, tacrolimus; LEF, leflunomide; RTX, rituximab; CsA, cyclosporine A.

treatment effect but also reduced hormone dosage and less resistance compared to traditional treatment regimen $(36,37)$. The present study compared three multi-target treatment regimens, namely the traditional regimen combined with RTX, the traditional regimen combined with LEF and the traditional regimen combined with TAC. The results suggest that the three multi-target treatment regimens are superior to the traditional treatment regimen and the ranking results suggested that the traditional regimen combined with TAC was the best of the nine regimens. It was also likely to have the lowest risk of infection in the treatment of RLN. Compared to traditional treatment regimen, multi-target therapy was not associated with any corresponding increase in adverse reactions. Furthermore, it not only produces an improved therapeutic effect but may also reduce the economic burden on patients to a certain extent (38). Thus, the clinical use of multi-target therapy should be promoted. However, with regard to the adverse reactions, including gastrointestinal reactions, infections and leukopenia, the multi-target therapy did not exhibit any difference from other regimens. However, this may be due to the small number of studies included.

In conclusion, the present study compared the effectiveness and safety of common therapeutic drugs and medication regimens in the treatment of $\mathrm{RLN}$. The results indicated the superiority of the regimen of $\mathrm{TAC}+\mathrm{CTX}+\mathrm{GC}$ regarding its clinical efficacy in patients with RLN. Furthermore, the traditional treatment regimen $(\mathrm{CTX}+\mathrm{GC})$ had the highest probability of causing adverse effects among the nine interventions compared. However, the present study had certain limitations. First of all, for most of the regimens, the data 
Table II. SUCRA values (\%) for treatment efficacy and safety of each regimen.

\begin{tabular}{lcccc}
\hline Treatment regimen & Efficacy & Infection & Gastrointestinal reaction & Leukocyte decline \\
\hline TAC+CTX+GC & 80.6 & 15.8 & 24.1 & N.A. \\
LEF+GC & 68.9 & N.A. & 23.3 & N.A. \\
CsA+GC & 65.9 & N.A. & N.A. & N.A. \\
RTX+CTX+GC & 64.3 & 55.0 & 59.5 & N.A. \\
TAC+GC & 54.8 & N.A. & 36.3 & 42.4 \\
LEF+CTX+GC & 54.1 & 69.1 & 79.1 & 59.0 \\
MMF+GC & 45.1 & 42.3 & 47.6 & 20.2 \\
CTX+GC & 12.7 & 67.8 & 80.1 & 65.4 \\
GC & 3.6 & N.A. & N.A. & 63.0
\end{tabular}

A greater SUCRA value indicates higher efficacy; thus, the effect of TAC+CTX+GC was indicated to be the best. A lower SUCRA value indicates a lower risk of adverse reactions; thus, the risk of infection with TAC+CTX+GC was the lowest. N.A. indicates failure to enter the analysis data and no corresponding value is provided. M/F, male/female; GC, glucocorticoid; CTX, cyclophosphamide; MMF, mycophenolate; TAC, tacrolimus; LEF, leflunomide; RTX, rituximab; CsA, cyclosporine A.

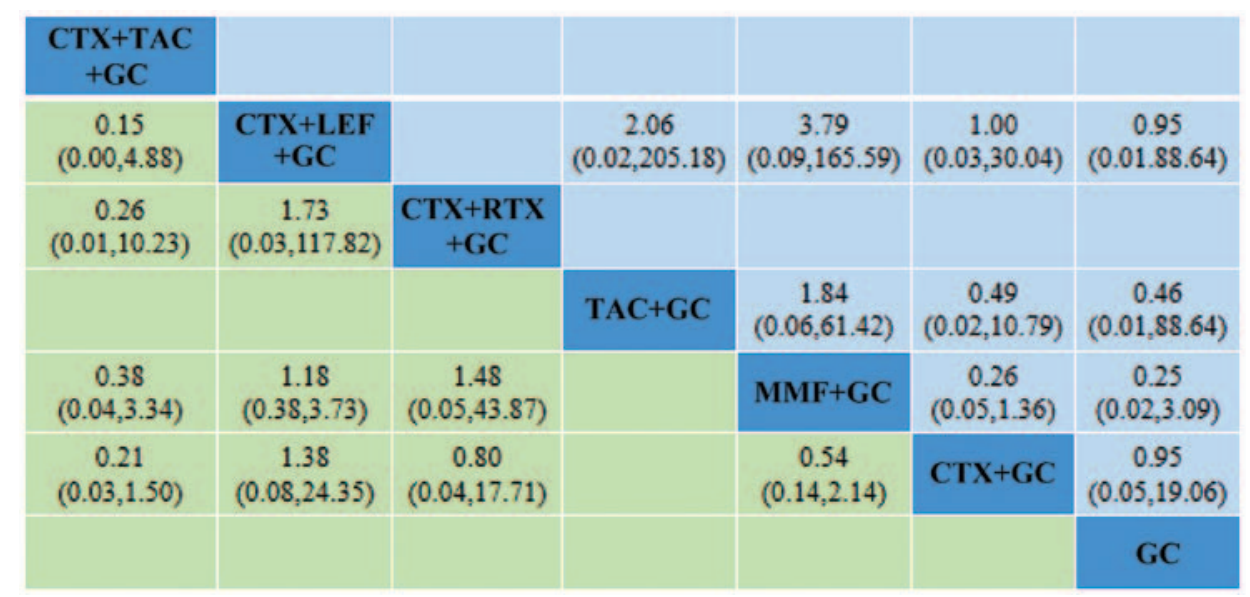

Figure 6. Comparison of gastrointestinal reactions and leukopenia after treatment with each regimen. Green indicates the comparison of gastrointestinal reactions after treatment with each regimen; light blue indicates the comparison of leukopenia after treatment with each regimen. GC, glucocorticoid; CTX, cyclophosphamide; MMF, mycophenolate; TAC, tacrolimus; LEF, leflunomide; RTX, rituximab; CsA, cyclosporine A.

for direct comparison were limited, resulting in insufficient evidence for indirect comparisons. Furthermore, previous studies have suggested that positive personal factors, including low-calorie diets and physical exercise, contribute to improved renal function $(39,40)$, but these factors were not considered in the present study and may have a potential impact on the results. Finally, in terms of adverse reactions, only infections, gastrointestinal reactions and leukopenia were analyzed, while other adverse reactions, including leukopenia, myelosuppression, alopecia, liver damage and menstrual disorders, were not analyzed due to incomplete data. Therefore, subsequent studies on RLN should focus on its adverse reactions when studying the curative effects.

\section{Acknowledgements}

The authors would like to thank Professor Dongqing Ye (Department of Epidemiology and Biostatistics, Anhui Medical University) for his encouragement and support.

\section{Funding}

This study was supported by the Natural Science Foundation of Anhui Province, China (grant nos. 1608085MH219 and 1808085QH251).

\section{Availability of data and materials}

The datasets used and/or analyzed during the current study are available from the corresponding author on reasonable request.

\section{Authors' contributions}

HY and HP conceived the study. MJT, LX and LRJ selected the studies. ZL and JS extracted the data. JZ analyzed the data and wrote the manuscript. All of the above authors contributed to the discussion of the results and revision of the manuscript. 


\section{Ethics approval and consent to participate}

Not applicable.

\section{Patient consent for publication}

Not applicable.

\section{Competing interests}

The authors declare that they have no competing interests.

\section{References}

1. Maroz N and Segal MS: Lupus nephritis and end-stage kidney disease. Am J Med Sci 346: 319-323, 2013.

2. Contis A, Vanquaethem H, Truchetet ME, Couzi L, Rigothier C, Richez C, Lazaro E and Duffau P: Analysis of the effectiveness and safety of rituximab in patients with refractory lupus nephritis: A chart review. Clin Rheumatol 35: 517-522, 2016.

3. Moroni G and Ponticelli C: The multifaceted aspects of refractory lupus nephritis. Expert Rev Clin Immunol 11: 281-288, 2015

4. Kronbichler A, Brezina B, Gauckler P, Quintana LF and Jayne DRW: Refractory lupus nephritis: When, why and how to treat. Autoimmun Rev 18: 510-518, 2019.

5. Momtaz M, Fayed A, Wadie M, Gamal SM, Ghoniem SA Sobhy N, Kamal Elden NM and Hamza WM: Retrospective analysis of nephritis response and renal outcome in a cohort of 928 Egyptian lupus nephritis patients: A university hospital experience. Lupus 26: 1564-1570, 2017.

6. Tang Y, Qin W, Peng W and Tao Y: Development and validation of a prediction score system in lupus nephritis. Medicine (Baltimore) 96: e8024, 2017.

7. The CL, Phui VE, Ling GR, Ngu LS, Wan SA and Tan CH: Causes and predictors of mortality in biopsy-proven lupus nephritis: The Sarawak experience. Clin Kidney J 11: 56-61, 2018.

8. Pons-Estel GJ, Serrano R, Plasín MA, Espinosa G and Cervera R Epidemiology and management of refractory lupus nephritis Autoimmun Rev 10: 655-663, 2011.

9. Xiang H: Clinical efficacy of leflunomide and cyclophosphamide in the treatment of patients with severe refractory lupus nephritis. Hebei Med J 39: 919-920, 2017 (In Chinese).

10. Jadad AR, Moore RA, Carroll D, Jenkinson C, Reynolds DJ, Gavaghan DJ and McQuay HJ: Assessing the quality of reports of randomized clinical trials: Is blinding necessary? Control Clin Trials 17: 1-12, 1996.

11. Zhang C, Yan JZ,Sun F,Liu Q, Guo Y andZeng XT:Differentiation and handling of homogeneity in network meta-analysis. Chin J Evidence Based Med 14: 884-888, 2014.

12. Ma Y, Song Y, Tong SS, Wang X, Wang GZ and Dai H: Analysis of the therapeutic effect of mycophenolate mofetil on refractory lupus nephritis. World Latest Med Information 16: 83, 2016 (In Chinese).

13. Li L: Analysis of the efficacy of mycophenolate mofetil in the treatment of refractory lupus nephritis and its effect on clinical symptoms. China Med Herald 9: 75-76, 2012 (In Chinese).

14. Wang Z, Zheng DW and Liu GH: The curative effect and safety of refractory lupus nephristis treated by mycophenolate mofetil combined with prednisone. J North Pharm 12: 127-128, 2015 (In Chinese)

15. Zheng S, Zheng JG, Han L and Wang DH: 31 cases of clinical curative effect analysis of mycophenolate mofetil treat refractory lupus nephritis. World Latest Med Information 15: 20-67, 2015 (In Chinese).

16. Dong YM, Zhou LZ, Li J, Li QH and Liu Z: Therapeutic effect of mycophenolate mofetil combined with prednisone in the treatment of refractory lupus nephritis. Chin J Clin Rational Drug Use 7: 44-45, 2014 (In Chinese).

17. Liu CY, Jiang Y and Wang L: Comparison of curative effect of mycophenolate mofetil and cyclophosphamide for the treatment of refractory lupus nephritis. China Modern Doctor 51: 48-50, 2013 (In Chinese).

18. Liu CY: Application of mycophenolate mofetil and cyclophosphamide in refractory lupus nephritis. Strait Pharmaceutical J 21: 137-138, 2009 (In Chinese).
19. Yang FF, Lin B, Wang J and You YW: Efficacy evaluation of combined immunosuppressive agents in the treatment of refractory lupus nephritis. Chin Youjiang Med J 36: 134-135, 2008 (In Chinese).

20. Zhen HM, Zhang HY, Huo G, Zhao SJ and Li JS: Clinical observation of steroid combined with mycophenolate mofetil in the treatment of refractory lupus nephritis. Inner Mongolia Med J 38: 1023-1025, 2006 (In Chinese).

21. Shi J, Chen BP and He XZ: Clinical efficacy of mycophenolate mofetil in the treatment of 26 cases of refractory type IV lupus nephritis. Negative 27: 1920, 2006 (In Chinese).

22. Li L, Li WQ and Qi WC: Clinical observation of mycophenolate mofetil in the treatment of refractory lupus nephritis. Chin J Postgraduates Med 25: 42-43, 2002 (In Chinese).

23. Lu Y: One-year follow-up observation of tacrolimus in the treatment of refractory lupus nephritis. J Med Forum 38: 167-169, 2017 (In Chinese).

24. Liu H, Huang DQ and Liang Y: Comparative study of tacrolimus and leflunomide in the treatment of refractory lupus nephritis. J Qiqihar Med University 37: 3419-3420, 2016 (In Chinese).

25. Liang B, Zhang JH, Wang CA and Han JL: Comparison of traditional treatment and multi-target treatment for refractory lupus nephritis. Chin J Control Endemic Diseases 29: 34-35, 2014 (In Chinese).

26. YI L, Luo FZ, Deng CP and Wu CY: Application of rituximab monoclonal antibody in the treatment of refractory lupus nephritis. J Nanjing Med University (Natural Sciences) 34: 1102-1104, 2014 (In Chinese).

27. Mostafi M, Rabbani MG, Hossain MR, Siddiqui AR and Rabbani SB: Cyclosporine \& mycophenolate mofetil in the treatment of cyclophosphamide refractory Class-IV lupus nephritis. JAFMC Bangladesh 5: 8-13, 2009.

28. Zhang J, Zhao $\mathrm{Z}$ and $\mathrm{Hu} \mathrm{X}$ : Effect of rituximab on serum levels of anti-C1q and antineutrophil cytoplasmic autoantibodies in refractory severe lupus nephritis. Cell Biochem Biophys 72: 197-201, 2015.

29. Mo YM and Li Long: Comparison of multiple target regimens and traditional classic regimens in the treatment of refractory lupus nephritis. Clin Misdiagnosis Mistherapy 22: 53-54, 2009 (In Chinese).

30. Kasitanon N, Boripatkosol P and Louthrenoo W: Response to combination of mycophenolate mofetil, cyclosporin A and corticosteroid treatment in lupus nephritis patients with persistent proteinuria. Int J Rheum Dis 21: 200-207, 2018.

31. Sedhain A, Hada R, Agrawal RK, Bhattarai GR and Baral A: Low dose mycophenolate mofetil versus cyclophosphamide in the induction therapy of lupus nephritis in Nepalese population: A randomized control trial. BMC Nephrol 19: 175, 2018.

32. Joo YB, Kang YM, Kim HA, Suh CH, Kim TJ, Park YW, Lee J, Lee JH, Yoo DH, Bae SC, et al: Outcome and predictors of renal survival in patients with lupus nephritis: Comparison between cyclophosphamide and mycophenolate mofetil. Int J Rheum Dis 21: 1031-1039, 2018

33. Hannah J, Casian A and D'Cruz D: Tacrolimus use in lupus nephritis: A systematic review and meta-analysis. Autoimmun Rev 15: 93-101, 2016.

34. Mok CC: Calcineurin inhibitors in systemic lupus erythematosus. Best Pract Res Clin Rheumatol 31: 429-438, 2017.

35. Bao H, Liu ZH, Xie HL, Hu WX, Zhang HT and Li LS: Successful treatment of class V+IV lupus nephritis with multitarget therapy. J Am Soc Nephrol 19: 2001-2010, 2008.

36. Jesus D, Rodrigues M, da Silva JAP and Inês L: Multitarget therapy of mycophenolate mofetil and cyclosporine A for induction treatment of refractory lupus nephritis. Lupus 27: 1358-1362, 2018.

37. Choi CB, Won S and Bae SC: Outcomes of multitarget therapy using mycophenolate mofetil and tacrolimus for refractory or relapsing lupus nephritis. Lupus 27: 1007-1011, 2018.

38. Xiao CJ, Rao H, Xu XY and Zeng QH: Clinical analysis of 23 cases of V+IV lupus nephritis treated with multiple targets. Chin J Clin (Electronic Edition) 7: 6733-6734, 2013.

39. Morales E, Valero MA, León M, Hernández E and Praga M: Beneficial effects of weight loss in overweight patients with chronic proteinuric nephropaties. Am J Kidney Dis 41: 319-327, 2003

40. Szulińska M, Skrypnik D, Ratajczak M, Karolkiewicz J, Madry E Musialik K, Walkowiak J, Jakubowski H and Bogdański P: Effects of endurance and endurance-strength exercise on renal function in abdominally obese women with renal hyperfiltration: A prospective randomized trial. Biomed Environ Sci 29: 706-771, 2016.

This work is licensed under a Creative Commons Attribution-NonCommercial-NoDerivatives 4.0 International (CC BY-NC-ND 4.0) License. 